\title{
Multiparametric Imaging for Presurgical Planning of Craniopagus Twins: The Experience of Two Tertiary Pediatric Hospitals with Six Sets of Twins
}

\author{
Karen A. Eley, FRCR, DPhil, MD* • Maria Camilla Rossi-Espagnet, MD* • Silvia Schievano, PhD • \\ Antonio Napolitano, PhD • Juling Ong, MBBS, FRCS • Aurelio Secinaro, MD • Luca Borro, MD • \\ David Dunaway, FRCS - Carlo Efisio Marras, MD - Adam Rennie, MD - Fergus Robertson, MD • \\ Sergio Picardo, MD • Daniela Longo, MD • Alessandro Inserra, MD • Massimo Rollo, MD • \\ Jessica Cooper, BSc • Kshitij Mankad, MBBS, FRCR • Noor-Owase Jeelani, MPhil, FRCS • Felice D'Arco, MD
}

\begin{abstract}
From the Department of Neuroradiology, Great Ormond Street Hospital for Children National Health Service Foundation Trust, London, England (K.A.E., A.R., F.R., J.C., K.M., F.D.); Department of Radiology, University of Cambridge School of Clinical Medicine, Box 218, Cambridge Biomedical Campus, Hills Road, Cambridge CB2 0QQ, England (K.A.E.); Neuroradiology Unit, Imaging Department, Bambino Gesù Children's Hospital, Rome, Italy (M.C.R.E., D.L., M.R.); Neuroscienze Salute Mentale e Organi di Senso Department, Sapienza University, Rome, Italy (M.C.R.E.); Department of Craniofacial Surgery, Great Ormond Street Hospital, London, England (S.S., J.O., D.D., N.O.J.); Advanced Cardiovascular Imaging Unit (A.S., L.B.), Department of General and Thoracic Surgery (A.I.), Medical Physics Department (A.N.), Neurosurgery Unit (C.E.M.), Bambino Gesù Children's Hospital, Scientific Institute for Research, Hospitalization and Healthcare, Rome, Italy; Department of Anesthesia and Critical Care, Ospedale Pediatrico Bambino Gesù, Rome, Italy (S.P.). Received May 19, 2020; revision requested June 30; revision received August 7; accepted August 28. Address correspondence to K.A.E. (e-mail: karen.a.eley@gmail.com).
\end{abstract}

${ }^{*}$ K.A.E. and M.C.R.E. contributed equally to this work.

Conflicts of interest are listed at the end of this article.

Radiology 2020; 00:1-10 • https://doi.org/10.1148/radiol.2020202216 • Content codes: CT MR PD

Conjoined twins are rare and pose a challenge to radiologists and surgeons. Craniopagus twins, where conjunction involves the cranium, are especially rare. Even in large pediatric centers, radiologists are unlikely to encounter more than one such event in their medical careers. This rarity makes it daunting to select a CT and MRI protocol for these infants. Using the experience of two tertiary pediatric hospitals with six sets of craniopagus twins, this multidisciplinary and multimodal integrated imaging approach highlights the key questions that need addressing in the decision-making process for possible surgical intervention.

() RSNA, 2020

Online supplemental material is available for this article.

$c$ onjoined twins are rare, with a prevalence of approximately 1.47 per 100000 births (1). Craniopagus twins are joined at any point of the cranium but not at the face or foramen magnum. This type represents approximately $2 \%-6 \%$ of all conjoined twins and occurs in approximately 0.6 per 1 million births, making it very rare in clinical practice and posing unique challenges to both radiologists and surgeons (2-4).

Interestingly, a female preponderance exists in craniopagus twins, with a female-to-male ratio of 4:1 (4). Antenatal diagnosis is possible with US at as early as 12 weeks gestation (5). Many sets of craniopagus twins do not survive to full term or to the immediate neonatal period, with only approximately $25 \%$ surviving to the point where surgical separation can be considered (3). However, surgical separation is becoming more successful because of advances in anesthesia and imaging. Lack of previous success was due to the reliance on older, conventional radiographic techniques, including pneumoencephalography (6). The multidisciplinary decision for surgery remains complex, as does the evaluation of the likelihood of success and the ethical dilemmas of potential adverse outcomes for one or both twins, including both high morbidity and mortality. This planning phase is typically an elective and prolonged process unless one of the twins dies. If this occurs, then expedient separation is necessary to prevent coagulopathy and related lethal complications in the surviving twin (7).
In this clinical context, multimodal imaging plays a paramount role in surgical planning by providing precise data on the complex anatomy of craniopagus twins. In the past 15 years, two of the largest European pediatric hospitals have evaluated six sets of craniopagus twins. Using this experience, we present a systematic approach to imaging acquisition and interpretation for craniopagus twins. This approach highlights the key questions that need to be addressed to assist the surgical teams in their decision-making process in possible twin separation.

\section{Imaging Planning}

Image acquisition in these patients requires careful preprocedural planning to ensure patient safety and good quality imaging while minimizing ionizing radiation exposure. A step-by-step approach should be carried out with attention to different aspects.

\section{Team Approach}

The most vital aspect is excellent communication between all members of the multidisciplinary team. Involved specialties will include radiology, neurosurgery, craniofacial surgery, anesthetics, pediatrics, three-dimensional (3D) printing technicians, physicists, allied nursing staff, and wider support services. Multidisciplinary team communication begins from the point of patient referral with 


\section{Abbreviation}

DSA = digital subtraction angiography, $3 \mathrm{D}=$ three-dimensional

\section{Summary}

Separation of craniopagus twins is a complicated procedure with ethical and technical considerations; a multimodal imaging presurgical assessment ensures comprehensive evaluation of the twins anatomy.

\section{Essentials}

- A multidisciplinary team is fundamental to guide presurgical diagnostic evaluation in craniopagus twins.

- A multimodal integrated imaging approach better defines complex extracranial and intracranial venous anatomy.

- Emerging imaging techniques, such as three-dimensional printing and virtual reality, represent useful surgical tools.

regular meetings regarding the status of the twins and planned hospital admission dates.

\section{Scanning Preparation}

A lead figure (radiographer or nurse) should be identified for both CT and MRI to undertake a full assessment of the logistics of the imaging investigations. This individual should also assess whether these procedures can be safely performed during one general anesthetic episode. Sufficient time should be allocated for scheduling to prepare the CT and MRI environments and to accommodate anesthetic time and any transfer between scanners. Because it is a rare occurrence to scan two patients simultaneously, the requirement for two sets of MRIcompatible monitoring and anesthetic equipment should not be overlooked.

For patients referred from elsewhere, previous images obtained at the referring center should be evaluated carefully in the immediate pre-imaging period. In our experience, available imaging is variable, from the most limited to comprehensive CT and/or MRI, prenatal US, and fetal MRI (8). Irrespective of this, we follow a standard imaging protocol for all sets of craniopagus twins on arrival because of the inevitable time delay in reaching our institutions.

The presumed duration of the protocols should be communicated to both anesthetists and nurses to optimize anesthetic administration. Moreover, a clear protocol outlining the order of acquisitions, contrast material doses, and the timing of contrast material administration will avoid confusion and error in a stressful environment.

\section{Team Briefing}

We follow the World Health Organization safety checklist and hold an initial team briefing before the twins' arrival to the radiology department (9).

\section{Limiting Access}

Imaging such rare and interesting patients will inevitably attract the attention of staff and students. As the console rooms are often confined spaces, access should be limited to key members of active staff; this must include the neuroradiologist during CT image acquisition to provide immediate confirmation of adequate imaging. We discourage other members of staff, such as the surgical team, other radiologists, and students, from being present at the time of CT and/or MRI scan acquisition.

\section{Anesthetics}

The anesthetic input for safe examination of the twins requires special mention. We use general anesthesia to ensure safety and to eliminate motion artifact. Prolonged sedation combined with transfer between CT and MRI is unlikely to be successful, except perhaps in the earliest neonatal period. This requires two anesthetic teams experienced in working in the MRI environment to independently care for each of the twins.

A reported potential problem during anesthesia is differential blood pressure between the twins because of the mixing of venous circulations. When positioning the twins for imaging, employing maneuvers, such as a gantry tilt, may provide a preferable solution to pharmacologic agents (10).

\section{Labeling and Orientation}

The key to accurate imaging interpretation relies on the correct labeling of twin 1 and twin 2. Decide before imaging how the twins should be oriented in the scanner. An olive oil capsule placed on the cheek of twin 1 is a useful reminder. This information should be recorded within the imaging descriptors to avoid errors at the time of reporting. The same orientation for both CT and MRI and for any subsequent imaging examination should be used. To avoid any potential discrepancy, all of the images should be stored preferably in both twins' imaging packets. Do not attempt to crop or rotate the image to the conventional head-up view for each twin unless explicitly annotated on the imaging headers.

The same labeling for each twin should be maintained throughout the entire hospital stay. A color-coding system may be helpful whereby each twin wears a specific color bracelet. This is matched with color coding of the individual teams caring for each twin during imaging and surgery. This system is extremely useful to avoid confusion among the staff and to remember the correct positioning of the twins.

\section{Imaging Protocols}

\section{Overview of Imaging Requirements}

For the surgical teams to plan surgical intervention and assess its feasibility, comprehensive imaging of the twins to delineate their combined anatomy is required. This shared anatomy will most often include the scalp, calvarium, meninges, and superficial venous drainage. Initial assessment includes CT examination with angiography and venography by means of sequential intravenous contrast material administration into each twin, with $3 \mathrm{D}$ reconstruction of the vessel anatomy and calvarium. MRI examination helps further assess meninges and brain parenchyma, and also maps eloquent areas, to determine the complexity of required surgery and potential adverse complications. Conventional digital subtraction angiography (DSA) provides detailed information on the arterial supply and venous drainage and serves to confirm the CT and MR angiographic findings (11).

CT and MRI protocols are summarized in Table 1 and Table 2, respectively. 


\section{Table 1: Suggested CT Protocols}

\begin{tabular}{ll}
\hline Parameter & Protocol \\
\hline Field of view & From vertex to C7 \\
Arterial phase & Bolus tracking with region of interest in internal carotid artery at C4 \\
Venous phase & 40 seconds after end of contrast material injection \\
Contrast material injection & $2 \mathrm{~mL} / \mathrm{kg}$ with $3 \mathrm{~mL} / \mathrm{sec}$ injection flow rate plus $12 \mathrm{~mL}$ saline chaser \\
\hline
\end{tabular}

Note.-For CT protocol, the whole protocol is acquired by contrast material injection into twin 1, allowing 30 minutes to elapse before contrast material injection into twin 2.

\begin{tabular}{|c|c|}
\hline Basic Sequence & Optional Sequence \\
\hline $\begin{array}{l}\text { Pre- and postcontrast 3D T1-weighted gradient echo (0.8-1-mm } \\
\text { slice thickness) }\end{array}$ & Diffusion tensor imaging \\
\hline $\begin{array}{l}\text { 3D T2-weighted fast spin echo (SPACE, Cube [GE Healthcare, } \\
\text { Milwaukee, Wis], VISTA) }(0.8-1-\mathrm{mm} \text { slice thickness) }\end{array}$ & Arterial spin labeling \\
\hline 3D FLAIR (0.8-1-mm slice thickness) & Time-resolved 3D angiography \\
\hline Axial diffusion-weighted imaging (3-mm slice thickness)* & 4D flow sequences \\
\hline \multicolumn{2}{|l|}{ Time of flight for MR angiography and venography } \\
\hline
\end{tabular}

CT protocol.-Multidetector imaging of the head and cervical spine should be performed, preferably with dual-source CT. Three-dimensional reconstructions with bone and soft-tissue algorithms are useful for planning plastic and craniofacial surgery to obtain anatomic information for skull separation and subcutaneous implantation of tissue expanders. These reconstructions are especially useful when studying the area of cranial fusion to estimate its extent and the spatial relation between the twins (Figs 1, 2).

CT angiography and venography are fundamental to exclude the presence of shared arterial vessels and to evaluate venous anatomy at the level of fusion. CT venography provides high-spatial-resolution images, which can be integrated with parenchymal MRI evaluation for presurgical modeling.

For CT angiography, we recommend administering $15 \%$ of the contrast material volume, waiting 2 minutes, and then using bolus tracking with manual scan initiation once bright contrast material fills the internal carotid artery lumen. This guarantees some peripheral enhancement and aids in the identification of areas of the brain of one twin perfused by the other (see Vascular Anatomy section). We use $2 \mathrm{~mL} / \mathrm{kg}$ of Omnipaque 350 (GE Healthcare, Cork, Ireland) or Iomeron 300 (Bracco Imaging, Milan, Italy) depending on patient age, with an injection flow rate of $3 \mathrm{~mL} / \mathrm{sec}$ whenever possible and a $12-\mathrm{mL}$ saline chaser. The dose per twin is calculated by dividing the combined weight of the twins in half. Venous imaging is performed after a period of about 40 seconds from the end of the contrast material injection. CT angiography and CT venography are then repeated in the same manner with injection into twin 2 after a delay of half an hour to allow for contrast material washout. We think precontrast images are not necessary, particularly with the use of dual-energy CT.

Multiplanar, maximum intensity projection images and $3 \mathrm{D}$ reconstructions should be produced at the end of the procedure.

MRI protocol.-MRI provides important information regarding the brain parenchymal anatomy, the tissue-vascular interface, meningeal defects, and associated malformations (Figs 3, 4). MRI data can be combined with results from CT angiography, venography, and DSA to obtain a more comprehensive evaluation of craniopagus twins anatomy with a particular focus at the site of union. In this region, it is paramount to obtain clear information about vascular (with CT angiography and venography) and parenchymal anatomy (with MRI with 3D sequences) and vascular flow dynamics (with DSA) (Fig 5).

For MRI acquisition, preplanning with a phantom is useful to determine the optimal coil coverage. We use a standard Siemens head phantom (Siemens, Erlangen, Germany) (5300 $\mathrm{mL}$ ) (composition [per $\left.1000 \mathrm{~g} \mathrm{H}_{2} \mathrm{O}\right] 3.75 \mathrm{~g} \mathrm{NiSO}_{4} \cdot 6 \mathrm{H}_{2} \mathrm{O}$ ). Surface coils cover the heads of both twins and have the added benefit of coil combination selection on some magnets. Standard head coils are not suitable in view of their closed superior aspect, which cannot accommodate the second twin.

A standard basic MRI protocol is suggested in Table 2.

High-spatial-resolution 3D volume acquisitions (slice thickness, $0.8-1 \mathrm{~mm}$ ) are preferred to allow for reconstruction in any plane because it is impossible to acquire imaging of both twins in the standard anatomic planes. Because the longest diameter of the twins is often in the $\mathrm{z}$ direction, imaging acquisition in the sagittal plane will save time for some sequences. 


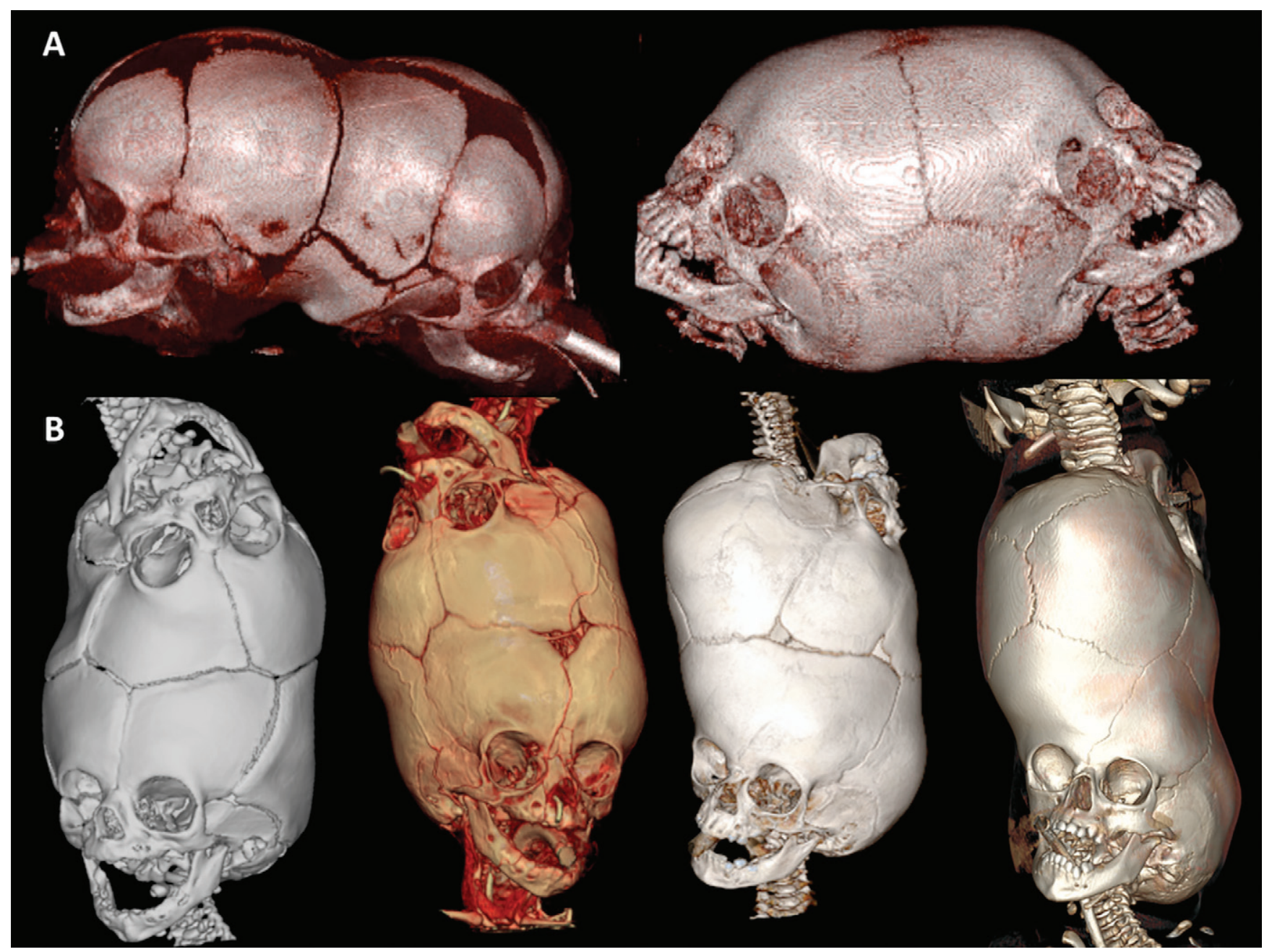

Figure 1: Volume-rendered CT images. A, Images in two sets of total craniopagus twins. Occipital craniopagus is shown on left, and vertical type 1 craniopagus is shown on right. B, Images in four sets of twins with total vertical craniopagus. Type 3 (intermediate rotation) vertical craniopagus is seen in first two twins at left and type 2 $\left(140^{\circ}-180^{\circ}\right.$ rotation) vertical craniopagus is seen in last two twins at right.

Postcontrast 3D T1-weighted gradient-echo isotropic imaging can be used to enhance visualization of complex venous anatomy and to permit surgical neuronavigation. In these specific gradient sequences, the venous sinuses appear filled with contrast material and appear as CT venogram-like images, which can be compared with MR venography and CT venography findings. Given the complex and shared venous anatomy, it is critical to have as much radiologic information about venous distribution as possible. MRI vascular sequences, such as time of flight and postcontrast dynamic angiography, help in the visualization of vascular, in particular venous, anatomy in relation to the brain parenchyma. These findings should be integrated with those obtained with CT venography and DSA to obtain a comprehensive view of the distorted anatomy that could be of great use to the neurosurgeons when deciding, for example, the site and trajectory of surgical access and when planning the different steps of separation to facilitate vascular flow adaptation.

In the same session, it is feasible to use more advanced techniques (Tables 1 and 2) to obtain information about white matter tract organization (with diffusion tensor imaging), parenchymal perfusion (with arterial spin labeling), vascular anatomy (with dynamic time-resolved 3D angiography with separate injections in both twins), and flow direction (with four-dimensional flow sequences [12]).

Conventional angiography. - Winston and colleagues $(13,14)$ first outlined the importance of assessing venous drainage in craniopagus twins for surgical planning in 1987. For this purpose, DSA still represents an essential technique for both anatomic delineation of arterial and venous anatomy and for dynamic evaluation of flow direction and vascular sharing between the twins (14). Digital angiography also plays a fundamental role in defining the efficacy of the surgical planned rerouting of venous circulation after the surgical procedure (15).

Selective DSA with catheterization of the internal and external carotid arteries and vertebral arteries is performed with delayed venous imaging by means of a sequential arterial puncture of each twin. This is performed on a separate occasion from CT and MRI, necessitating a further anesthetic procedure. DSA allows for the identification of arterial circulation sharing, for confirmation of previously obtained CT and MRI data. It also provides dynamic evaluation of venous circulation 

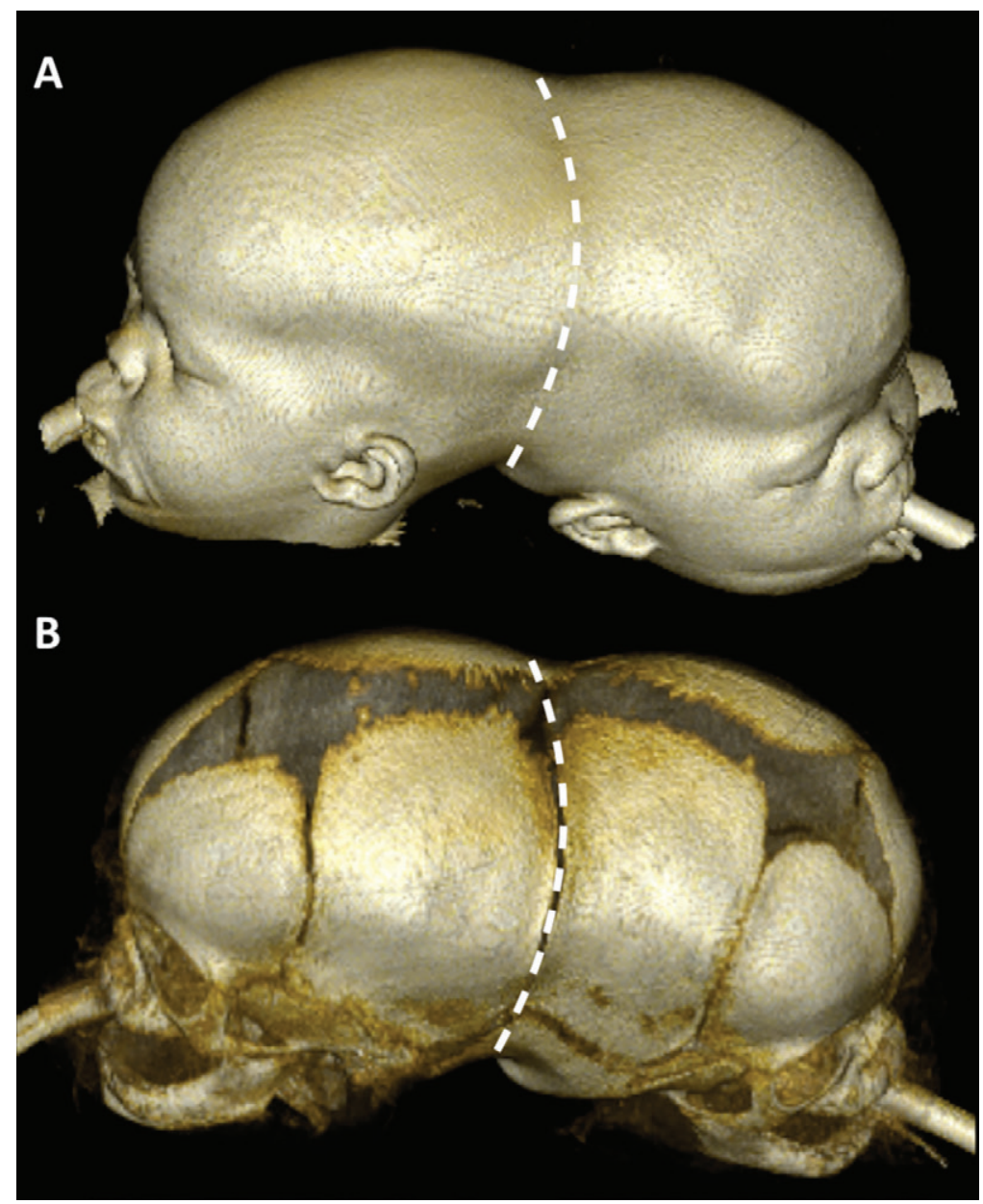

Figure 2: Volume-rendered unenhanced $C T$ images can be useful for, $A$, scalp and, $B$, bone anatomy definition to plan plastic surgical reconstruction. Area of contact (dotted line) can be measured to estimate volume of tissue expansion required.

and helps in the identification of the main routes of drainage in each twin, information that is fundamental to plan each surgical step. The integration of other imaging data, particularly from MRI, is fundamental in identifying relationships between vascular anatomy (obtained with higher spatial and temporal resolution with DSA) and parenchymal anatomy (illustrated with MRI) (Fig 5, C, D). In select cases, DSA enables the possibility to assist surgery in vascular separation using embolization of communicating vessels (11).

\section{Key Findings of Imaging Interpretation}

\section{Craniopagus Classification}

The orientation of the twins will have been ascertained clinically. CT imaging (Fig 1) will confirm the site of cranial union. The degree of bony union and availability of bone stock should be assessed for future skull reconstruction of the twins, including the presence of diploic bone and the presence of paranasal sinuses in older children.

The literature describes a variety of craniopagus classification systems, with the O'Connell classification being the first reported in 1976 (2), and probably the most used, especially after the revision made by Stone and Goodrich in 2006 (3). The
O'Connell classification is based on the size of the union and extracranial versus intracranial involvement (2-4).

The intracranial contents should be assessed to determine if there is any sharing of the meninges or vasculature. This provides the most basic distinction into partial or total craniopagus.

In partial types, the less common variant, the area of contact between the two skulls is small, and the intertwin longitudinal axis may be less than $140^{\circ}$ in the angular form (frontal, occipital, or parietal) or between $140^{\circ}$ and $180^{\circ}$ in the vertical variant. In partial craniopagus twins, a bony septum may separate the two brains, the leptomeninges are usually separate and have little or no vascular sharing, and the calvarial convexity is largely preserved $(3,16,17)$.

In comparison, total craniopagus refers to those with more extensive sharing of intracranial structures (4). The cerebral arterial supply is generally separate from minor leptomeningeal connections, although larger arteries may occasionally cross from one twin to the other (16). The cerebral venous drainage is generally shared with the superior sagittal sinus replaced by a complete or incomplete circumferential venous sinus, as more frequently observed in total vertical craniopagus twins. Substantial mixing of the venous circulations occurs, and it is common for the blood to drain preferentially to one twin (16).

The orientation of the twins' faces provides further subclassification, as follows: type 1 faces the same direction, type 2 faces the opposite direction $\left(140^{\circ}-180^{\circ}\right)$, and type 3 has an intermediate angle of rotation $(16,18)$ (Fig 1$)$.

Multiple further subdivisions of these basic classifications are described in the literature (4). We recommend providing 3D-reconstructed imaging of the scalp together with the bone to assist the surgeons (Figs 2, E1 [online]).

\section{Parenchyma}

The distortion of parenchymal anatomy can make orientation and interpretation challenging. Three-dimensional volume imaging with multiplanar reconstruction is of great assistance. The dura should be traced methodically to determine which areas have a clear cleavage plane between the two brains and which lobes of the twins are in contact (Fig 3). The examination should focus on areas of dural defects at the twin interface. In one of the sets of twins, we attempted slice-by-slice segmentation (using both T2- and T1-weighted images) of the dura with subsequent $3 \mathrm{D}$ printing in three different colors indicating "presence of dura," "possible presence of dura," and "absence of dura." This proved useful for presurgical planning (Movie 1 [online]).

The development of the falx cerebri along the midline during fetal life is thought to influence the correct development of the dural venous sinuses and of the superior sagittal sinus (15). Thus, the visualization of the meninges and of the falx in craniopagus twins may indirectly indicate the disposition of the sinuses (15). 


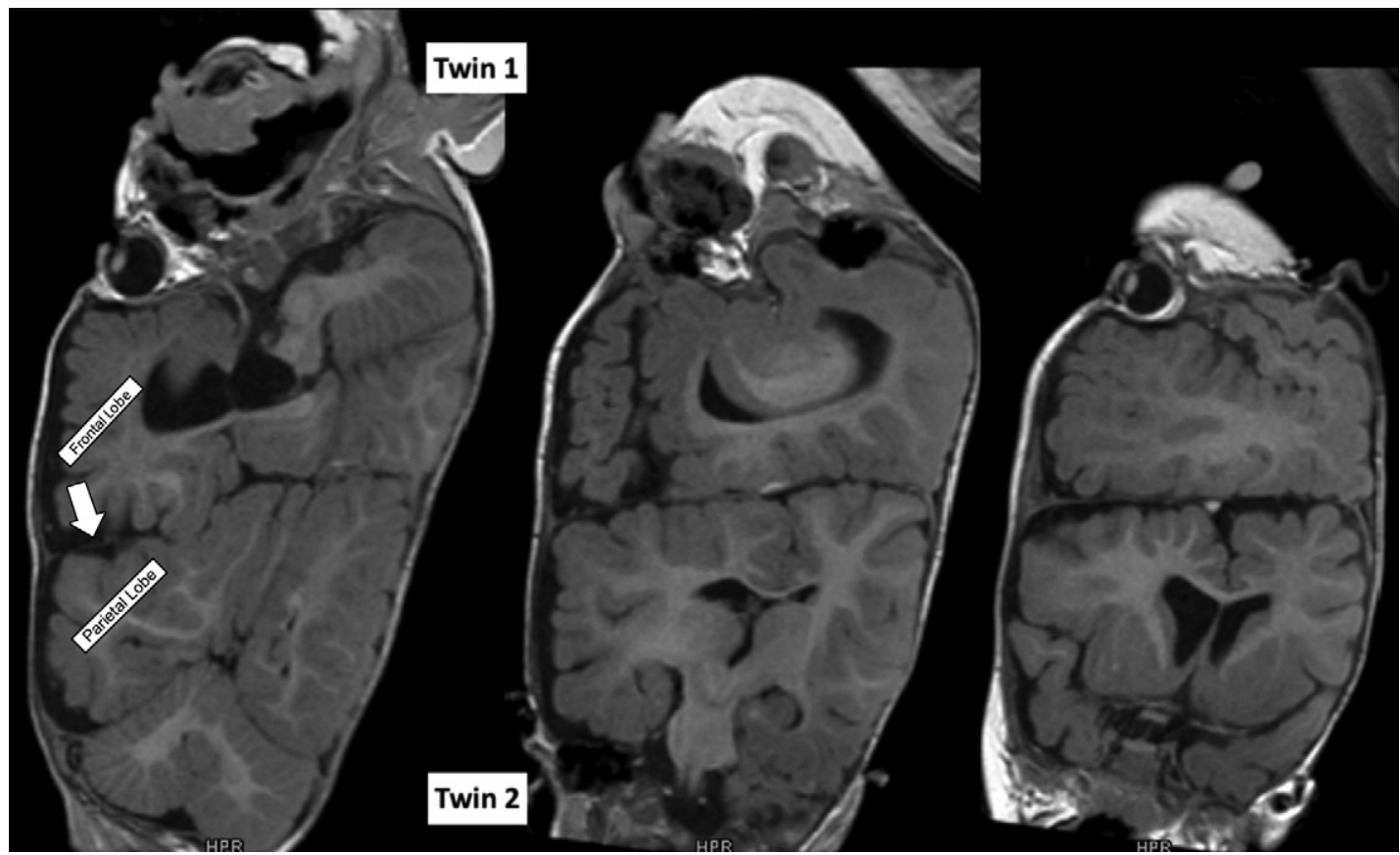

Figure 3: Unenhanced T 1 -weighted volume images in craniopagus twins. Twin 1 is at the top of all three images, and twin 2 is at the bottom of all three images. Each twin was imaged in turn to identify lobes (which are often distorted) and dura and to determine sites of contact. Images were obtained from left to right in the sagittal plane in twin 1 and from posterior to anterior in coronal plane in twin 2. Superior surface of twin I's left frontal lobe lies opposite the lateral convexity surface of twin 2's right parietal lobe, and dura (arrow) in this section is mostly intact.

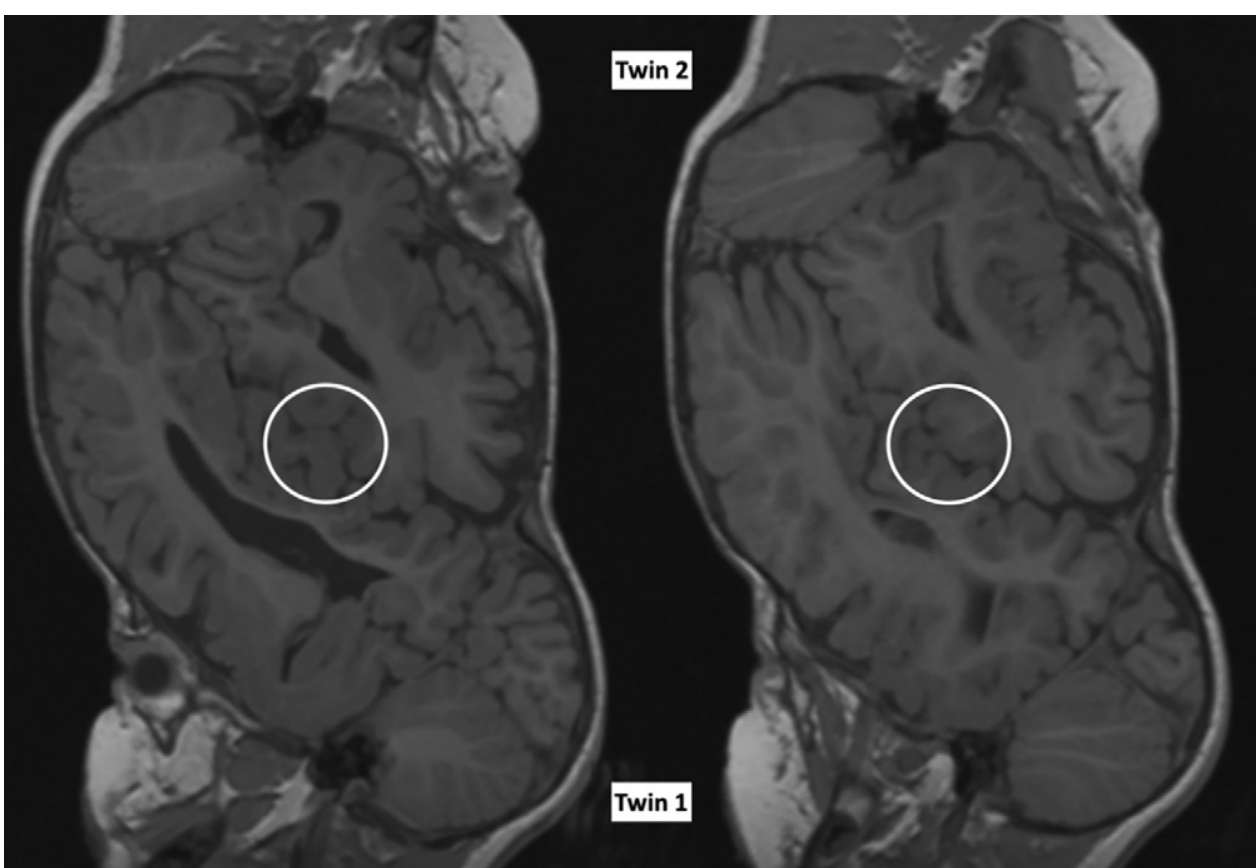

Figure 4: Unenhanced T1-weighted volume images illustrate examples of parenchymal bridging between right parietal lobe of twin 1 and right parietofrontal lobe of twin 2 (circles). Dura should be carefully traced to identify defects and parenchymal bridging. For each area of bridging, the anatomic segment involved for both twins should be determined (allowing for distorted anatomy) along with whether this involves eloquent areas. This requires identification of central sulcus, which may not be clearly identified.

Investigators should assess whether any small parenchymal bridges exist between the two brains where no apparent cleavage plane or dura is visualized (Fig 4) and document the involved parts of the brain for both twins. If these parts include any eloquent regions, those should be highlighted. Further assessment can be performed with supplemental imaging techniques such as arterial spin labeling or diffusion tensor imaging. Functional MRI can be considered in older children (who are able to follow necessary commands) to define hemispheric language dominance (19). Advanced MRI sequences can be technically challenging in craniopagus twins (eg, arterial spin labeling) or create problems in interpretation because of the distorted anatomy (eg, color mapping in diffusion tensor imaging that will not respect the standard color coding). Thus, we suggest using and interpreting them with extreme caution. Using the CT angiography acquisition methods described earlier can depict the leptomeningeal vascularization with clarity, giving a perfusion-like image to identify part of the brain of one twin vascularized by the other (Fig 5). 


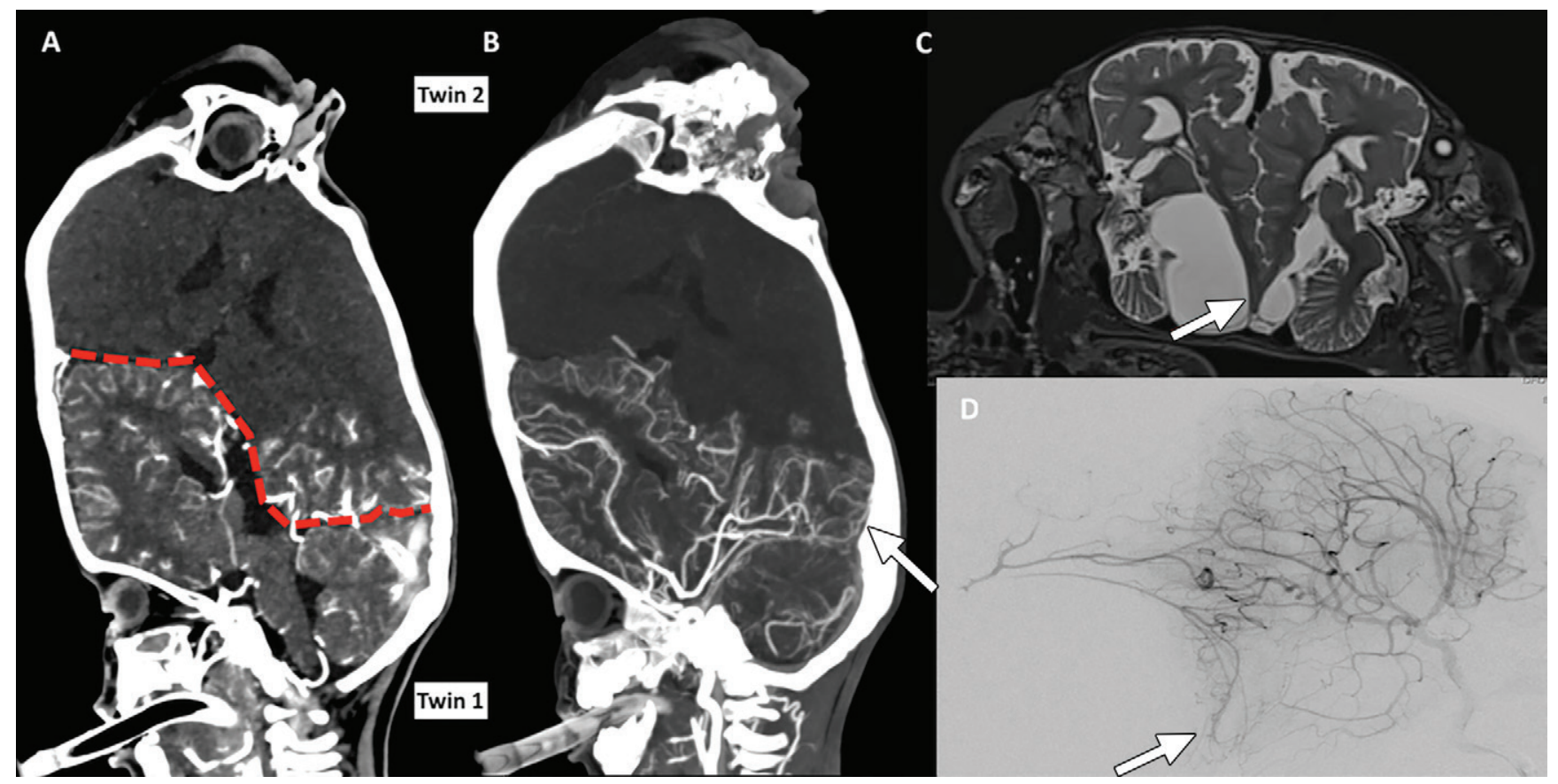

Figure 5: Images show the use of combined information from MRI and CT imaging to assess arterial supply of each brain with sequential contrast material injection into each twin at CT angiography. Findings are confirmed with conventional angiography. A, B, Maximum intensity projection CT angiograms. The dashed line in A highlights boundary between the two brains to demonstrate that parietal lobe of twin 2 receives arterial supply from twin 1 . The same perfused territory was seen in twin 1 when twin 2 was injected with contrast material (arrow in B). In both twins, dominant supply to posterior parietal lobe was from posterior cerebral artery of other twin, with collateral supply from their own anterior cerebral artery. C, Sagittal unenhanced three-dimensional T2-weighted image (repetition time msec/echo time msec, 3200/378) demonstrates presence of small connection between occipital lobes of twins (arrow). D, Image from digital subtraction angiography confirms the findings in $C$, showing connection between small occipital leptomeningeal arteries (arrow).

There should be a full review of the brain parenchyma to assess for any associated malformations, diffusion abnormalities, and the myelination status. The ventricles and basal cisterns should be assessed before moving on to the craniocervical junction and imaged cervical spine.

\section{Vascular Anatomy}

CT angiographic, CT venographic, MR angiographic, and MR venographic imaging for any shared arterial supply or venous drainage should be assessed. Subsequent DSA of both carotid arteries and vertebrobasilar systems should be performed in both twins (20) to corroborate the findings at CT and MRI (Fig 5).

First, the arterial system should be assessed for any cross-over of supply between the twins by carefully reviewing CT imaging following injection of a contrast medium into each twin sequentially. Correlating the vascular imaging with the predetermined anatomic layout of the two brains will assist in ascertaining the location of any shared arterial supply (Fig 5). Minor leptomeningeal connections are often observed, and larger arteries may occasionally cross from one twin to the other (17). Maximum intensity projection reconstructions are often useful in determining the origin of such vessels. The characteristics of the cerebral venous system are an important prognostic indicator for successful separation (4).

Second, the deep venous system, which is often normal or less affected, should be evaluated.

Third, the dural venous sinuses, which are usually abnormal, should be evaluated. These malformations range from partial connections, where only small parts of the sinuses are shared by means of anastomotic communications, to complete absence of major sinuses and collateral superficial shared vessels that account for the main venous drainage (Figs 6, E2, F, G [online]). Dural venous sinuses develop in regions of dural reflections. Thus, they will be absent when the dura mater is defective $(16,21)$. The superior sagittal sinuses can be fused at the midpoint with large anastomosis, and part of the drainage can be provided with a complete or incomplete circumferential venous sinus (Figs E2, $F, G$ [online]). Investigators should determine, as much as possible, which twin has the circumferential venous sinus and how much of the venous drainage from the second twin enters this, and from which areas of the brain. The presence of any smaller collateral venous drainage pathways should be ascertained.

The vascular anatomy will provide insight into the extent of venous reconstruction required and the areas of potential ischemia and sacrifice. Endovascular techniques have been reported in the literature, including temporary balloon occlusion before vessel sacrifice and coil embolization (16); however, multistage surgical separation is usually preferred.

\section{Imaging Review, Postprocessing, and 3D Models}

We encourage independent neuroradiologic review of images before collaborative rereview and discussion with one consensus report. Reviewing the images with the surgical teams is vital in highlighting key findings.

The production of anatomic models will provide additional understanding of any shared anatomy, but this requires careful imaging segmentation, which can be both challenging and 


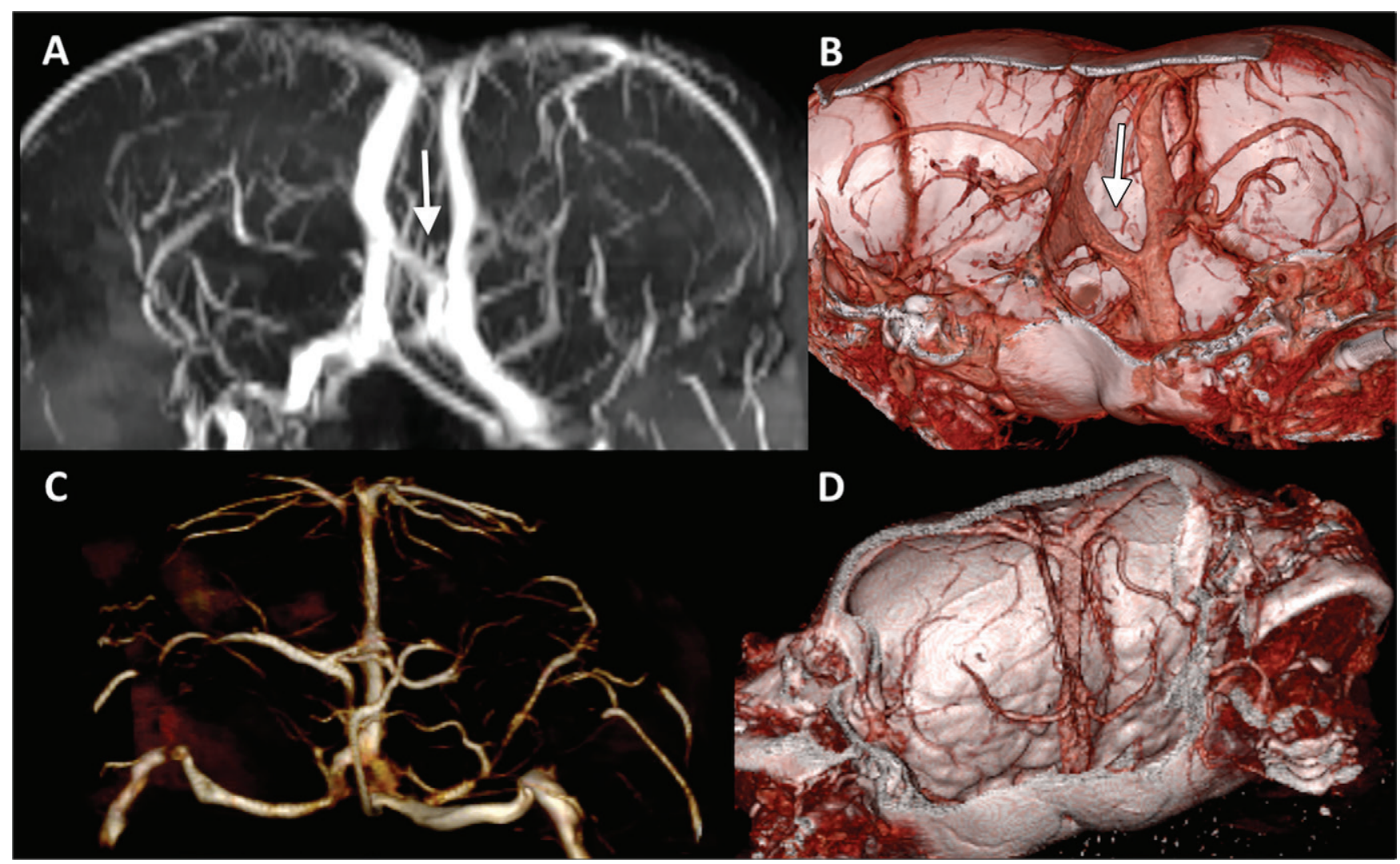

Figure 6: $\quad A, C, M R$ angiograms and, $B, D, C T$ venograms illustrate how the combined information is useful for surgical planning in craniopagus twins to assess the relationship between dural venous sinuses with brain and bone, respectively, as correct definition of dural sinus anatomy can be challenging. This anatomy can vary from small venous bridges (arrow in $A$ and $B$ ) to sharing of entire sinuses or tracts like in these craniopagus twins, where they share posterior part of superior sagittal sinus (as seen in $C$ and $D$ ).

time consuming. Image processing for our cases was performed with Mimics software (version 23; Materialise, Leuven, Belgium). Close collaboration between the $3 \mathrm{D}$ printing technicians and radiologists ensures that the segmentation process is an accurate representation of the anatomy. Once the imaging data set has been segmented, it is optimized with a software such as Meshmixer (version 3.5; Autodesk, San Rafael, Calif), where supporting struts between free-floating anatomic structures can be inserted as necessary. Commercial 3D printers are now able to print complex anatomic models from a range of materials, such as biocompatible thermopolymers (ie, polylactic acid) in multiple colors (22).

Newer methods such as virtual 3D imaging permit visualization of anatomy in a more immersive way, allowing for further comprehension of the relationships of the structures (Movies 1, 2 [online]).

In addition, advanced MRI techniques and postprocessing tools, such as four-dimensional flow imaging, help integrate the imaging data to visualize and quantify venous flow orientation and drainage (23), particularly for complex dural connections in craniopagus twins. Venous flow volume directions and quantification provide additional information about the hemodynamic interactions between the two connected dural systems. Interpretation of this data must be validated, but additional four-dimensional flow calculations can be used to better guide intraoperative management in specific circumstances (Fig 7).

\section{Associated Anomalies}

Finally, craniopagus twins frequently present with additional comorbidities, including cardiovascular, genitourinary, craniofacial, and neurologic abnormalities (4), occurring in up to $79 \%$ of reported craniopagus twins. The increased venous drainage to one twin can result in a higher cardiac and urine output and hypertension in this twin (17). It is therefore imperative that a full assessment is made of the twins by general pediatricians, who should provide overarching care and coordination of any additional investigations.

\section{Reporting}

A report example is provided in Appendix E1 (online).

The report should be divided into the following sections: (a) introduction, where the radiologic labeling is described and the type of craniopagus deformity with orientation of twin 1 to twin 2 is clearly stated; (b) skull and bone appearances and relationships; (c) brain parenchyma appearances and relationships; and (d) vascular anatomy. It is useful for clinicians and surgeons to have relevant screenshots embedded in the report (Fig E2 [online]) to help interpret the description of complex radiologic findings. It can often be difficult to identify the perirolandic regions because of the spatial distortion of the lobes, but if the twins are younger than 1 year, then the myelinated tracts can be traced back to the eloquent cortex areas (Fig E2 [online]). 

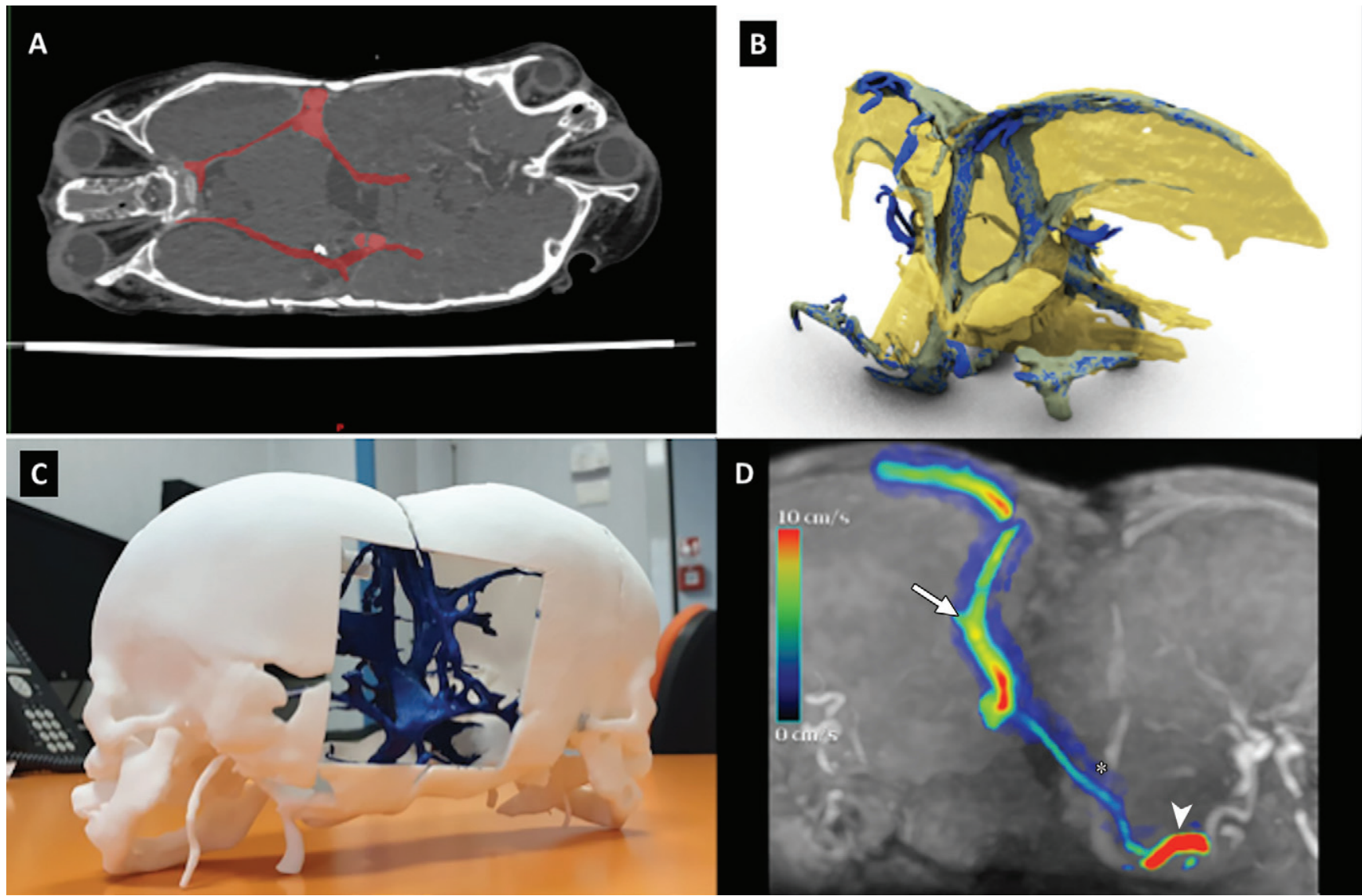

Figure 7: Examples of three-dimensional (3D)-printed anatomic models and images obtained with advanced techniques used for surgical planning. A, Axial postcontrast CT image in craniopagus twins. Images in the venous phase were processed with semiautomatic threshold algorithm using range of attenuation values between 978 $\mathrm{HU}$ and $1768 \mathrm{HU}$. Segmentation was performed using Mimics 3D segmentation software (version 23; Materialise, Leuven, Belgium). B, The 3D rendering is transferred to 3D rendering web viewer platform (Sketchfab, New York, NY), which allows surgical team to explore and navigate 3D model using a web page, with powerful rendering capability. C, "In-house" 3D printing of model was carried out with fused deposition modeling 3D printer using white biocompatible material (polylactic acid). To differentiate anatomic structures, venous vessels were colored in blue. D, Advanced MRI techniques, such as four-dimensional flow sequences, can also be exploited to obtain information on flow velocity. In this case, intracranial veins obtained with a sagittal four-dimensional flow sequence are segmented with dedicated software (CMR42, version 5. 12; Circle Cardiovascular Imaging, Calgary, Canada). Posterior portion of superior sagittal vein of twin A (arrow), torcular venous connection (*), and dominant transverse sinus of twin B (arrowhead) are shown in flow velocity color-coded scale.

\section{Surgical Considerations}

The primary goal of surgery in craniopagus twins is to accomplish a correct separation by minimizing the morbidity and mortality mostly related to the complexity and degree of dural venous sinus sharing that may influence and cause intraoperative bleeding, ischemia, and venous hypertension, leading to brain edema (3). Moreover, cerebrospinal fluid leakage, meningitis, seizures, and hydrocephalus may represent severe postoperative complications $(13,24)$. Therefore, in 2016, Harvey et al (4) reviewed all reports of separation or attempts at separation of craniopagus twins in the past 20 years (from 1995 to 2015) to identify the factors that may influence a successful separation. Separation was attempted in 14 twin pairs, with success in nine. Only one variable among those investigated (classification, shared cerebral venous drainage, sex, age, and comorbidities) was associated with successful separation: the presence of vertical craniopagus. However, successful separation was observed with staged separation (where surgery is completed in multiple discrete procedures) and age of separation ( $<12$ months).
A staged (multiple surgeries) versus a nonstaged (single surgery) procedure is thought to be the preferred surgical approach. Drummond et al (25) initially proposed the staged procedure with the primary aim of separating a shared superior sagittal sinus. A staged procedure includes a period of tissue expansion (for subsequent scalp closure) and sequential surgeries to separate the vasculature and parenchyma. This gradual separation encourages the development of collateral circulation draining to the deep venous system in the twin losing the circumferential venous sinus, allowing for gradual adaptation of both twins to a major alteration in venous drainage (25). Multiple staged surgeries have the advantage of reducing the time of each surgical procedure, thus minimizing the exposure to anesthesia and lowering the risk of complications such as bleeding, edema, and ischemia (26). The interval between surgical stages may allow for vascular changes such as the development of collateral flow and time for physical therapy (27). Follow-up imaging of the staged separation is often necessary to assess expected and unexpected complications. 


\section{Conclusion}

Providing all imaging information to the surgical team permits effective discussion regarding the potential technical and ethical problems of surgical separation (28). The feasibility of separation must be weighed against temporary or permanent harm or death of one or both twins. Failure to separate the twins is likely to condemn them to a difficult future.

In conclusion, radiology plays a vital role in the preoperative assessment and surgical planning of craniopagus twins. Successful imaging acquisition depends on careful planning and communication, with special attention made to correct labeling and imaging orientation both at the time of acquisition and during imaging interpretation.

Acknowledgments: This study was supported by the Gemini Untwined Study Group, which includes Richard Hayward, Yean Chooi, Alessandro Borghi, Deborah Zeitlin, Kar-binh Ong, Sally Wilmshurst, Dominic Thompson, Mario Zama, Lorenzo Figà-Talamanca, Andrea Carai, Sergio Giombini, Enrico Castelli, Luigi Zucaro, Martina Lucignani, Anna Contini, Fabio Marconi, Tom Minden, Laura Sinclair, Zubair Tahir, Bridget Callahan, Lara Van De Lande, Cecelia Carney, Greg James, and Jane Herod, and the Great Ormond Street Hospital occupational and physiotherapy team, which includes Camila Maglaya-Ocampo, Bridget Callahan, Endrit Pajaziti, Ellis Clarke, and Neil Bulstrode.

We thank the families of the patients.

We also thank the senior co-authors, Noor-Owase Jeelani, David Dunaway, and Kshitij Mankad, for their clinical care, surgical planning, and expert contributions to this paper.

We dedicate this paper to James Goodrich, MD, PhD (1946-2020), a pediatric neurosurgeon and pioneer in the surgical separation of craniopagus twins.

Author contributions: Guarantors of integrity of entire study, K.A.E., M.C.R.E., F.D.; study concepts/study design or data acquisition or data analysis/interpretation, all authors; manuscript drafting or manuscript revision for important intellectual content, all authors; approval of final version of submitted manuscript, all authors; agrees to ensure any questions related to the work are appropriately resolved, all authors; literature research, K.A.E., M.C.R.E., A.S., D.D., S.P., A.I., M.R., K.M., N.O.J., F.D.; clinical studies, K.A.E., M.C.R.E., A.S., D.D., C.E.M., F.R., S.P., A.I., F.D., M.R., J.C., K.M., N.O.J.; experimental studies, S.S., A.S., L.B., S.P.; and manuscript editing, K.A.E., M.C.R.E., A.N., J.O., A.S., L.B., D.D., C.E.M., A.R., F.R., S.P., D.L., A.I., M.R., J.C., K.M., N.O.J., F.D.

Disclosures of Conflicts of Interest: K.A.E. disclosed no relevant relationships. M.C.R.E. disclosed no relevant relationships. S.S. Activities related to the present article: institution received European Research Council Starting Grant. Activities not related to the present article: is a consultant for Medtronic Cardiovascular Structural Team and Gore Cardiovascular; has grants/grants pending with British Heart Foundation, National Institute for Health Research Biomedical Research Center, and La Fondation Dassault Systèmes; has patents planned, pending, or issued. Other relationships: disclosed no relevant relationships. A.N. disclosed no relevant relationships. J.O. disclosed no relevant relationships. A.S. Activities related to the present article: disclosed no relevant relationships. Activities not related to the present article: has received payment for lectures, including service on speakers bureaus, from Siemens and Bracco. Other relationships: disclosed no relevant relationships. L.B. disclosed no relevant relationships. D.D. disclosed no relevant relationships. C.E.M. disclosed no relevant relationships. A.R. disclosed no relevant relationships. F.R. disclosed no relevant relationships. S.P. disclosed no relevant relationships. D.L. disclosed no relevant relationships. A.I. disclosed no relevant relationships. M.R. disclosed no relevant relationships. J.C. disclosed no relevant relationships. K.M. Activities related to the present article: disclosed no relevant relationships. Activities not related to the present article: provides expert testimony. Other relationships: disclosed no relevant relationships. N.O.J. disclosed no relevant relationships. F.D. disclosed no relevant relationships.

\section{References}

1. Mutchinick OM,Luna-MuñozL, AmarE, et al. Conjoined twins: a worldwide collaborative epidemiological study of the International Clearinghouse for Birth Defects Surveillance and Research. Am J Med Genet C Semin Med Genet 2011;157C(4):274-287.

2. O'Connell JEA. Craniopagus twins: surgical anatomy and embryology and their implications. J Neurol Neurosurg Psychiatry 1976;39(1):1-22.

3. Stone JL, Goodrich JT. The craniopagus malformation: classification and implications for surgical separation. Brain 2006;129(Pt 5):1084-1095.

4. Harvey DJ, Totonchi A, Gosain AK. Separation of craniopagus twins over the past 20 years: A systematic review of the variables that lead to successful separation. Plast Reconstr Surg 2016;138(1):190-200.

5. Mahale A, Venugopal A, Kishore MS, Ghiraiya A. Craniopagus: a preoperative assessment. Indian J Radiol Imaging 2006;16(4):783-784.

6. Duboulay GH. Radiological examination of two pairs of craniopagus twins. BMJ 1964;1(5394):1337-1338.

7. Nejat F, Habibi Z, Goudarzi M, et al. Emergency separation of craniopagus twins: case report. J Neurosurg Pediatr 2017;20(4):307-313.

8. Goldman-Yassen AE, Goodrich JT, Miller TS, Farinhas JM. Preoperative Evaluation of Craniopagus Twins: Anatomy, Imaging Techniques, and Surgical Management. AJNR Am J Neuroradiol 2020;41(6):951-959.

9. Haynes AB, Weiser TG, Berry WR, et al. A surgical safety checklist to reduce morbidity and mortality in a global population. $\mathrm{N}$ Engl J Med 2009;360(5):491-499.

10. Rutka JT, Souweidane M, ter Brugge K, et al. Separation of craniopagus twins in the era of modern neuroimaging, interventional neuroradiology, and frameless stereotaxy. Childs Nerv Syst 2004;20(8-9):587-592.

11. Parameswari A, Vakamudi M, Raghupathy V, Siddhartha R. Anaesthetic management of total craniopagus twins for magnetic resonance imaging and cerebral angiography. Br J Anaesth 2010;105(3):368-370.

12. Holmgren M, Wåhlin A, Dunås T, Malm J, Eklund A. Assessment of Cerebral Blood Flow Pulsatility and Cerebral Arterial Compliance With 4D Flow MRI. J Magn Reson Imaging 2020;51(5):1516-1525.

13. Winston KR, Rockoff MA, Mulliken JB, Strand RD, Murray JE. Surgical division of craniopagi. Neurosurgery 1987;21(6):782-791.

14. Winston KR. Craniopagi: anatomical characteristics and classification. Neurosurgery 1987;21(6):769-781.

15. Lasjaunias P, Kwok R, Goh P, Yeong KY, Lim W, Chng SM. Adevelopmental theory of the superior sagittal sinus(es) in craniopagus twins. Childs Nery Syst 2004;20(8-9):526-537.

16. Dunaway D, Jeelani NUO. Staged separation of craniopagus twins. Semin Pediatr Surg 2015;24(5):241-248.

17. Stanley P, Anderson FM, Segall HD. Radiologic investigation of craniopagus twins (partial type). AJNR Am J Neuroradiol 1983;4(2):206-208.

18. Sudha L, Dev B, Kamble R, Joseph S. Role of biplane digital subtraction angiography, and $3 \mathrm{D}$ rotational angiography in craniopagus twins: A case report, detailed pictorial evaluation, and review ofliterature. J Pediatr Neurosci 2009;4(2):113-116.

19. Ho YCL, Goh KYC, Golay X, et al. Functional magnetic resonance imaging in adult craniopagus for presurgical evaluation. J Neurosurg 2005;103(5):910916.

20. Schindler E, Hajek P. Craniopagus twins: neuroradiological findings (CT, angiography, MRI). Neuroradiology 1988;30(1):11-16.

21. Gupta DK, Mahapatra AK; AIIMS Team Craniopagus. Addressing the Shared Circumferential Sinus in Craniopagus Conjoined Twins: Is Venous Bypass an Option? World Neurosurg 2020;133:421-422.

22. Eley KA. Centralised 3D printing in the NHS: a radiological review. Clin Radiol 2017;72(4):269-275.

23. Rivera-Rivera LA, Johnson KM, Turski PA, Wieben O. Pressure mapping and hemodynamic assessment of intracranial dural sinuses and dural arteriovenous fistulas with 4D flow MRI. AJNR Am J Neuroradiol 2018;39(3):485-487.

24. Frazee J, Fried I, Kawamoto H, et al. The separation of Guatemalan craniopagus twins. Childs Nerv Syst 2004;20(8-9):593-600.

25. Drummond G, Scott P, Mackay D, Lipschitz R. Separation of the Baragwanath craniopagus twins. Br J Plast Surg 1991;44(1):49-52.

26. Staffenberg DA, Goodrich JT. Separation of craniopagus conjoined twins: an evolution in thought. Clin Plast Surg 2005;32(1):25-34, viii.

27. Staffenberg DA, Goodrich JT. Separation of craniopagus conjoined twins with a staged approach. J Craniofac Surg 2012;23(7 Suppl 1):2004-2010.

28. Lee M, Gosain AK, Becker D. The bioethics of separating conjoined twins in plastic surgery. Plast Reconstr Surg 2011;128(4):328e-334e. 